\title{
Effective potential and vacuum stability
}

\author{
Vincenzo Branchinat \\ Department of Physics, University of Catania and \\ INFN, Sezione di Catania, Via Santa Sofia 64, I-95123, Catania, Italy \\ Hugo Faivre2, Vincent Pangon 3 \\ IPHC/DRS, Theory Group - Louis Pasteur University and CNRS, \\ 23 rue du Loess, 67037 Strasbourg, France
}

\section{Abstract}

By following previous work on this subject, we investigate the issue of the instability of the electroweak vacuum against the top loop corrections by performing an accurate analysis of a Higgs-Yukawa model. We find that, when the physical cutoff is properly implemented in the theory, the potential does not exhibit any instability. Moreover, contrary to recent claims, we show that this instability cannot be understood in terms of the very insightful work of $\mathrm{Wu}$ and Weinberg on the non-convexity of the one-loop effective potential of a scalar theory. Some of the theoretical and phenomenological consequences of our results are briefly discussed.

\section{Introduction}

It is commonly believed that the top loop contribution to the Higgs effective potential $V_{\text {eff }}(\phi)$ destabilizes the electroweak (EW) vacuum [1. According to our recent analysis, however, this instability results from extrapolating $V_{\text {eff }}(\phi)$ beyond its region of validity [2]. When we limit ourselves to the range of $\phi$ where the approximations considered for its computation hold, $V_{\text {eff }}(\phi)$ turns out to be a convex (therefore, a fortiori, stable) function of its argument, in agreement with well known exact theorems [3, 4].

In order to avoid unnecessary complications related to the gauge sector of the Standard Model (SM), in [2] we started our analysis by considering a Higgs-Yukawa model. In fact, for large values of the Yukawa coupling, this model presents the same (apparent) instability of the SM.

Our work has been recently challenged in 5 and this provides one of the motivations for the present paper. By pursuing our investigation on this issue, we shall see that some of the arguments considered in [5] are incorrect, while some others do not apply to the

\footnotetext{
${ }^{1}$ vincenzo.branchina@ct.infn.it

${ }^{2}$ hugo.faivre@ires.in2p3.fr

${ }^{3}$ vincent.pangon@ires.in2p3.fr
} 
problem under investigation. The results of our present analysis, in fact, reinforce the conclusions of our previous work: the vacuum instability is unphysical and is nothing but the result of an illegal extrapolation of the computed potential beyond its region of validity.

Let us consider the Higgs-Yukawa model:

$$
\mathcal{L}(\phi, \psi, \bar{\psi})=\frac{1}{2} \partial_{\mu} \phi \partial_{\mu} \phi+\bar{\psi} \gamma_{\mu} \partial_{\mu} \psi+\frac{m_{\Lambda}^{2}}{2} \phi^{2}+\frac{\lambda_{\Lambda}}{24} \phi^{4}+g_{\Lambda} \phi \bar{\psi} \psi,
$$

where $\Lambda$ is the ultraviolet cutoff of the theory and $m_{\Lambda}^{2}, \lambda_{\Lambda}$ and $g_{\Lambda}$ are the bare mass and coupling constants respectively. The bare potential for the scalar field is:

$$
V_{\Lambda}(\phi)=\frac{m_{\Lambda}^{2}}{2} \phi^{2}+\frac{\lambda_{\Lambda}}{24} \phi^{4}
$$

A straightforward computation of the one-loop effective potential $V_{1 l}^{(Y)}(\phi)$ gives the well known result (see, for instance, [2]):

$V_{1 l}^{(Y)}(\phi)=\frac{m^{2}}{2} \phi^{2}+\frac{\lambda}{24} \phi^{4}+\frac{\left(m^{2}+\frac{\lambda}{2} \phi^{2}\right)^{2}}{64 \pi^{2}}\left(\ln \left(\frac{m^{2}+\frac{\lambda}{2} \phi^{2}}{\mu^{2}}\right)-\frac{3}{2}\right)-\frac{g^{4} \phi^{4}}{16 \pi^{2}}\left(\ln \frac{g^{2} \phi^{2}}{\mu^{2}}-\frac{3}{2}\right)$,

where $m^{2} \equiv m_{\mu}^{2}, \lambda \equiv \lambda_{\mu}, g \equiv g_{\mu}$ and $\mu$ is the renormalization scale.

With the help of renormalization group ( $R G$ ) techniques, it is possible to improve on this result [6]. For the purposes of our analysis, however, we can limit ourselves to consider the one-loop approximation to $V_{\text {eff }}(\phi)$, as the RG-improvement has no relevance to our discussion (see [2] for details).

We see from Eq.(3) that, when the Yukawa coupling is sufficiently strong, $V_{1 l}^{(Y)}(\phi)$ becomes unstable for large values of $\phi$. Although this is at odds with the convexity property of the exact $V_{\text {eff }}(\phi)$, it is argued in [5] that $V_{1 l}^{(Y)}(\phi)$ can be trusted even in the region where it is not convex. The authors base their reasoning on the analysis presented in [7] (see also [8]), where the issues of the physical meaning of the imaginary part of the one-loop effective potential $V_{1 l}^{(s)}(\phi)$ of the self-interacting scalar theory alone (i.e. the theory obtained from Eq.(1) for a vanishing Yukawa coupling) and the non-convexity of its real part were addressed.

As we shall explain in the following (see section 2), although physically meaningful and relevant to the issues considered in that paper, the analysis put forward in [7] bears no relation with the problem of the instability induced by the fermion loop contribution to $V_{1 l}^{(Y)}(\phi)$. In fact, it turns out that the origin of the non-convexity of $V_{1 l}^{(Y)}(\phi)$ in the region of large $\phi$, which is our problem, is totally different from the origin of the non-convexity of $V_{1 l}^{(s)}(\phi)$ in the region between the classical minima, which is the problem studied in [7].

\footnotetext{
${ }^{4}$ We use the notation $V_{1 l}^{(Y)}(\phi)$ to indicate the Higgs-Yukawa one-loop effective potential, while the one-loop potential of the scalar theory alone will be indicated in the following with $V_{1 l}^{(s)}(\phi)$.
} 
The interpretation found in [7] for the non convex part of $V_{1 l}^{(s)}(\phi)$ cannot be extended to the non-convexity of $V_{1 l}^{(Y)}(\phi)$.

The paper is organized as follows. In section 2, we begin by briefly reviewing the analysis of 7 on the interpretation of the non-convex and non-real parts of the oneloop approximation to the effective potential $V_{1 l}^{(s)}(\phi)$ of the scalar self-interacting theory. Then, we show why, contrary to recent claims [5], these results bear no relation with the instability shown by the Higgs-Yukawa one-loop potential $V_{1 l}^{(Y)}(\phi)$. In section 3, we show that the region of $\phi$ where the renormalized Higgs-Yukawa one-loop potential apparently becomes unstable lies beyond the region of validity of perturbation theory. In section 4 , we draw our conclusions.

\section{Instability in the internal and in the external regions}

For the purposes of our analysis, it is worth to recall the following definition of the effective potential $V_{\text {eff }}(\phi)$ for a single component scalar theory. Given the Hamiltonian density $\hat{\mathcal{H}}$, $V_{\text {eff }}(\phi)$ is obtained by minimizing the expectation value of $\hat{\mathcal{H}}$,

$$
V_{\text {eff }}(\phi)=\min \langle\Psi|\hat{\mathcal{H}}| \Psi\rangle
$$

with the constraints,

$$
\langle\Psi \mid \Psi\rangle=1 \quad \text { and } \quad\langle\Psi|\hat{\phi}| \Psi\rangle=\phi .
$$

In order to support the reliability of the instability induced by the fermion loop contribution to $V_{1 l}^{(Y)}(\phi)$, the authors of [5] invoke the work of Wu and Weinberg [7]. However, we now show that the analysis presented in [7], although very illuminating on the physical meaning of $V_{1 l}^{(s)}(\phi)$ (the one-loop effective potential of a single component scalar selfinteracting theory), has nothing to do with the instability problem under investigation. To this end, let us first briefly review the main results of [7].

In this work, the authors consider a single component scalar theory which exhibits symmetry breaking at the classical level. As is well known, $V_{1 l}^{(s)}(\phi)$ develops an imaginary part for values of $\phi$ between the inflection points of the potential. The aim of [7] is to investigate the physical meaning of the real and imaginary parts of $V_{1 l}^{(s)}(\phi)$. After a thorough analysis, they come to the conclusion that the function that $V_{1 l}^{(s)}(\phi)$ is approximating is not $V_{\text {eff }}(\phi)$ as defined by Eqs.(4) and (5), but a sort of modified effective potential, which they call $\widetilde{V}(\phi)$, obtained by requiring that the state $|\Psi\rangle$ (i.e. the wave functional $\Psi[\phi]=\langle\phi \mid \Psi\rangle$ ) be localised 5 around $\phi$. The real part of $V_{1 l}(\phi) \simeq \tilde{V}(\phi)$ (see Fig(1) turns out to be the expectation value of the energy density on such a localized (unstable) state while its imaginary part is half its decay rate per unit volume [7].

As for $V_{\text {eff }}(\phi)$, the true effective potential, it turns out that in the region between the classical minima $-v$ and $+v$ (the so called internal region) it is well approximated

\footnotetext{
${ }^{5}$ This question was already discussed by P.M. Stevenson in 9] in the framework of the Gaussian approximation to the effective potential.
} 


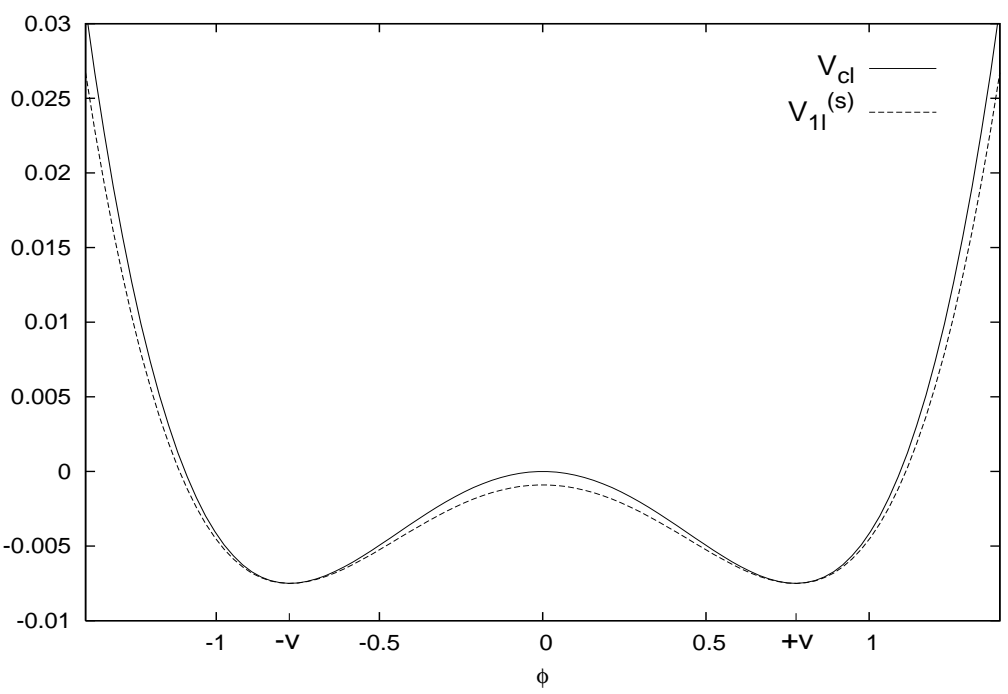

Figure 1: The continuous line represents the classical potential $V_{c l}(\phi)$ of a single component scalar theory in the broken phase, while the dashed line is the real part of the one-loop effective potential $V_{1 l}^{(s)}(\phi)$. The latter provides a good approximation for the real part of $\widetilde{V}(\phi)$, the modified effective potential for localized states (see text).

by the Maxwell construction, whereas in the region where $|\phi| \geq v$ (the external region) $V_{1 l}(\phi)$ provides a good approximation to $V_{\text {eff }}(\phi)$ [7, 10] (see Fig,2). Actually, for any given value of $\phi$ in the internal region, the true vacuum state of the system is given by an inhomogeneous mixed state which is a linear combination of $|+v\rangle$ and $|-v\rangle$ [7].

Within the framework of the loop expansion, the origin of the Maxwell construction can be easily understood once we realize that in the internal region the one-loop approximation, which is based on the tacit assumption that the path integral is dominated by a single saddle point, looses its validity. For those values of $\phi$, there are two competing non trivial saddle points which contribute with the same weight to the path integral. Taking into account the contribution of both of them, the flat shape of $V_{\text {eff }}(\phi)$ between $-v$ and $+v$ (Fig [2) immediately arises [10]. This also explains why, in the external region, the usual loop expansion, in particular the one-loop approximation $V_{1 l}(\phi)$, provides a good approximation to $V_{e f f}(\phi)$. In fact, for those values of $\phi$, there is no competition of saddle points; the path integral is saturated by a single saddle point and the usual loop expansion is at work.

Having reviewed the main results of [7], now we can see why this work (although interesting for the insight it provides on the physical understanding of $V_{1 l}^{(s)}(\phi)$ ) bears no relation with (therefore, cannot be invoked to support the reliability of) the instability induced by the fermion loop corrections in the one-loop effective potential $V_{1 l}^{(Y)}(\phi)$ of the Higgs-Yukawa model.

We begin by noting that this instability (non-convexity) occurs in the external region (see Fig, 3), the region beyond the minima, while the analysis of [7] concerns the non- 


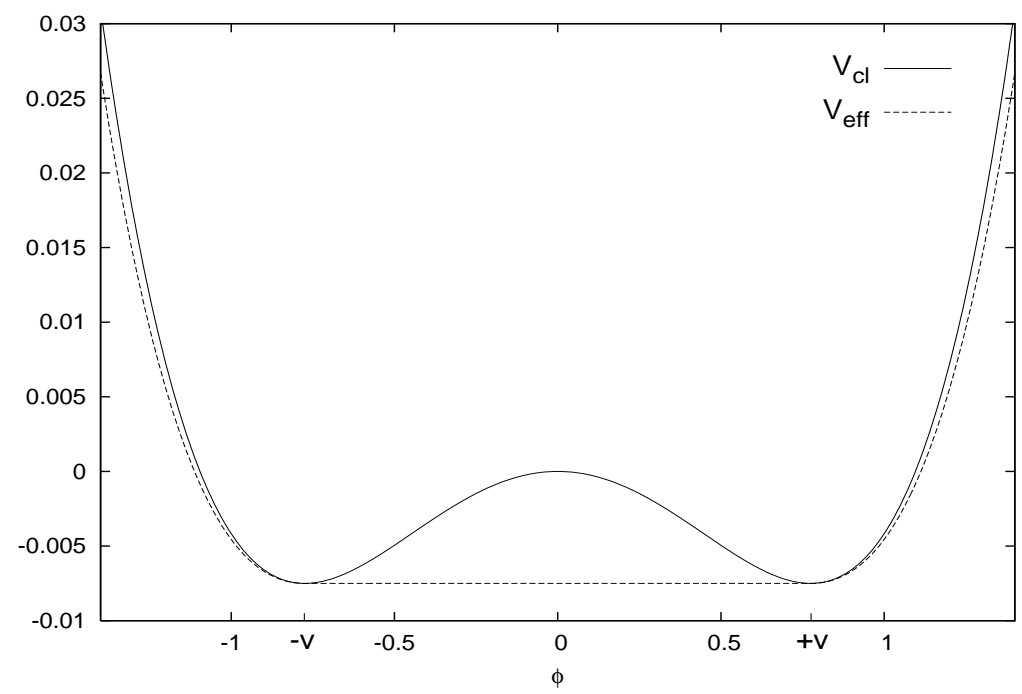

Figure 2: The continous line represents the classical potential $V_{c l}(\phi)$ of a single component scalar theory in the broken phase, while the dashed line is the effective potential $V_{\text {eff }}(\phi)$. In the internal region (i.e. in the region between the classical minima), $V_{\text {eff }}(\phi)$ is well approximated by the Maxwell construction (internal dashed line). In the external region it is well approximated by $V_{1 l}^{(s)}(\phi)$ (external dashed line). As is clear from the figure, this approximation of $V_{\text {eff }}(\phi)$ is a convex function of $\phi$.

convexity of the effective potential in the internal region (see Fig, 2), the region between the minima. Moreover, the instability in our problem results from the integration over the fermion modes and this has nothing to do with the internal region non-convexity studied in [7, which results from the competition of two non trivial saddle points with the same weight. It is then clear that the external region non-convexity of $V_{1 l}^{(Y)}(\phi)$ has a totally different origin from the internal region non-convexity.

The fact that, for sufficiently large values of the field, $V_{1 l}^{(Y)}(\phi)$ bends down is due to the negative contribution of the fermion loop, the term $-g^{4} \phi^{4} \ln \phi^{2}$ of Eq.(3)), which overwhelms the classical $\lambda \phi^{4}$ term for large values of $\phi$. Obviously, this has nothing to do with the non-convexity in the internal region, which comes from the presence of a negative $\mathrm{m}^{2}$. In this respect, it is worth to note that the external region instability of $V_{1 l}^{(Y)}(\phi)$ occurs even for positive values of $m^{2}$ (see Fig.3).

We are now in the position to draw our first conclusion. Although at this stage of our analysis we do not know yet whether the instability of $V_{1 l}^{(Y)}(\phi)$ is a physical effect or not, we have already established that (contrary to what is claimed in [5]) it is not possible to invoke the fact that the non-convexity of $V_{1 l}^{(s)}(\phi)$ in the internal region has a sensible physical interpretation, which is the result of [7], to argue that the instability of $V_{1 l}^{(Y)}(\phi)$ in the external region is a genuine physical effect.

Therefore, the physically relevant question to ask at this point is the following. Can we give a physically meaningful interpretation to the non-convex part of $V_{1 l}^{(Y)}(\phi)$ in the 


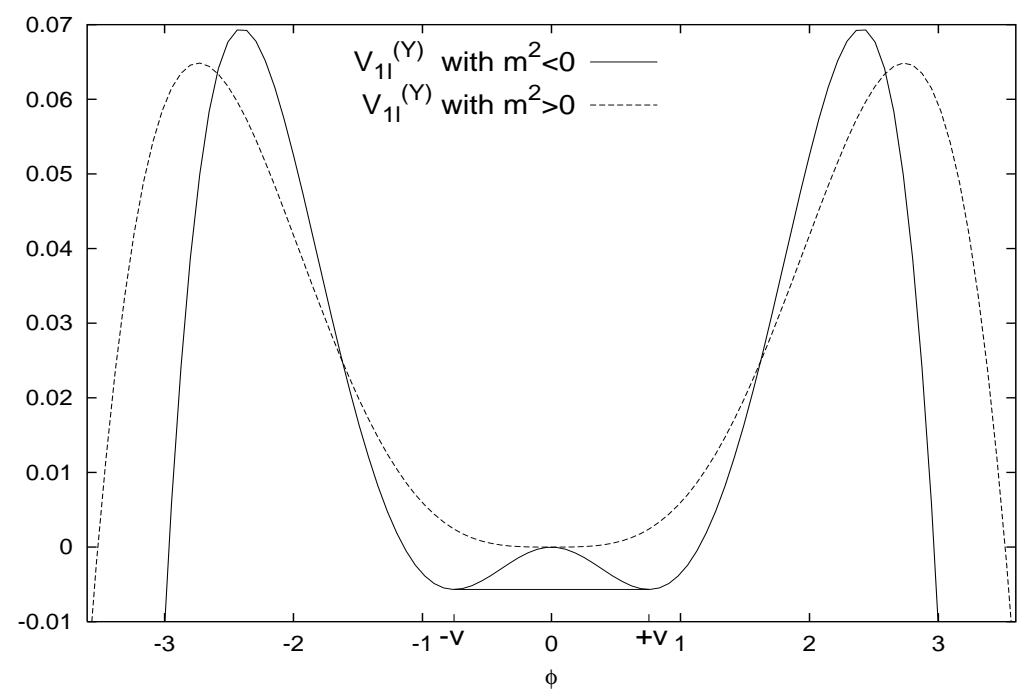

Figure 3: Continous line: the renormalized one-loop potential $V_{1 l}^{(Y)}(\phi)$ of the HiggsYukawa model as given in Eq.(3) $\left(m^{2}<0\right)$. The (apparent) instability occurs in the external region, i.e. for $|\phi|>+v$, far away from the internal region where the competition of two saddle points with the same weight gives rise to the Maxwell construction, the horizontal line between $-v$ and $+v$ (see text). Dotted line: $V_{1 l}^{(Y)}(\phi)$ for a positive value of $m^{2}$. As noted in the text, while in the internal region the one-loop potential is now convex, the apparent instability in the external region still persists. This figure clearly shows the different origin of the non-convexity in the internal and external regions.

external region (Fig, 3) as we can do for the non-convexity in the internal one [7]? In the following section we shall find that, in this region, the approximations used for the computation of the renormalized $V_{1 l}^{(Y)}(\phi)$ break down. Moreover, we shall see that, when the cutoff is properly implemented in the theory, no instability occurs.

\section{Instability within perturbation theory and RG}

Going back to the renormalized Higgs-Yukawa potential $V_{1 l}^{(Y)}(\phi)$ of Eq.(3), we see that the instability is driven by the fermion contribution, which is also the dominant correction term. Therefore, with no loss of generality, we can neglect in this equation the boson contribution, as well as other unimportant finite terms, so that the one-loop potential takes the (simplified) form:

$$
V_{1 l}^{(Y)}(\phi) \simeq \frac{m_{\mu}^{2}}{2} \phi^{2}+\frac{\lambda_{\mu}}{24} \phi^{4}-\frac{g^{4} \phi^{4}}{16 \pi^{2}} \ln \frac{\phi^{2}}{\mu^{2}}
$$

The first two terms correspond to the classical potential $V_{c l}(\phi)$, which is written in terms of the IR parameters $m_{\mu}^{2}$ and $\lambda_{\mu}$, with $m_{\mu}^{2}<0$ and $\lambda_{\mu}>0$. The potential is typically 
normalized so that

$$
V_{1 l}^{(Y)}( \pm v)=0
$$

where $\pm v$ are the classical minima. Then, the instability takes place when $V_{1 l}^{(Y)}(\phi)$ becomes negative. Note also that, as the instability occurs for large values of $\phi$, in the following discussion we can further simplify Eq.(6) by neglecting the mass term and write:

$$
V_{1 l}^{(Y)}(\phi) \simeq \frac{\lambda_{\mu}}{24} \phi^{4}-\frac{g^{4} \phi^{4}}{16 \pi^{2}} \ln \frac{\phi^{2}}{\mu^{2}} .
$$

A point which is relevant to our analysis concerns the sign of $\lambda_{\Lambda}$. In [5] is said that there is no reason why it should be positive, and we would like to turn our attention to this issue. First we note that, with the help of simple renormalization group analysis, the effective potential in Eq.(8) can be written as:

$$
V_{1 l}^{(Y)}(\phi) \simeq \frac{\lambda_{\mu}}{24} \phi^{4}-\frac{g^{4} \phi^{4}}{16 \pi^{2}} \ln \frac{\phi^{2}}{\mu^{2}}=\frac{\lambda_{\Lambda}}{24} \phi^{4}+\frac{g^{4} \phi^{4}}{16 \pi^{2}} \ln \frac{\Lambda^{2}}{\phi^{2}} .
$$

Eq.(9) tells us that, if $\lambda_{\Lambda}$ is positive, $V_{1 l}^{(Y)}(\phi)$ is also positive, at least in the region where it is defined, i.e. for $\phi<\Lambda$. Therefore, from Eq.(7) we see that no instability can occur.

Now, it is not difficult to convince ourselves that $\lambda_{\Lambda}$ is positive. From a physical point of view, in fact, the theory has to be regarded as an effective theory, intrinsically defined with a cutoff $\Lambda$, the higher energy scale where it is still valid. The bare potential, which defines the theory at the scale $\Lambda$, has to be well behaved. For a negative value of $\lambda_{\Lambda}$, however, we would have a potential unbounded from below and the theory would not be defined.

Moreover, we observe that, within the framework of cutoff perturbation theory, in order to get the $-g^{4} \phi^{4} \ln \left(\phi^{2} / \mu^{2}\right)$ term, which is responsible for the instability of $V_{1 l}^{(Y)}(\phi)$ in Eq.(3), we have to consider the counterterm $\frac{\delta \lambda}{24} \phi^{4}$, where:

$$
\frac{\delta \lambda}{24}=-\frac{g^{4}}{16 \pi^{2}} \ln \frac{\Lambda^{2}}{\mu^{2}}
$$

Let us assume now that a range of $\phi$ 's exists such that $V_{1 l}^{(Y)}(\phi)<0$ and that in this region $V_{1 l}^{(Y)}(\phi)$ can be trusted (so that we can claim instability of the potential). As:

$$
\Lambda>\phi
$$

we immediately have $V_{1 l}^{(Y)}(\Lambda)<V_{1 l}^{(Y)}(\phi)<0$, which combined with Eq.(9) gives:

$$
\frac{\lambda_{\Lambda}}{24}=\frac{\lambda_{\mu}}{24}-\frac{g^{4}}{16 \pi^{2}} \ln \frac{\Lambda^{2}}{\mu^{2}}<0 .
$$

Being the renormalized quartic coupling constant $\lambda_{\mu}$ positive, which is a necessary condition to have a non trivial classical minimum, the result $\lambda_{\Lambda}<0$ implies that (the absolute 
value of) $\delta \lambda$ must be greater than $\lambda_{\mu}$ itself, which would signal a breakdown of perturbation theory.

Finally, we note that the result $\lambda_{\Lambda}=\lambda_{\mu}+\delta \lambda$, with $\delta \lambda$ given by Eq.(10), is nothing but the result that would be obtained with the help of the perturbative RG equation for the running coupling constant $\lambda(p)$ at $p=\Lambda$. Therefore, it is easy to rephrase the above considerations in a renormalization group language. The apparent instability of the effective potential is related to the fact that the RG equations would drive $\lambda_{\mu}$ to negative values in the high energy regime (see, for instance, [1]). As we have just seen, however, this range of energies is not allowed.

In this respect, we note that an interesting discussion, which closely parallels and complements our results, can be found in [11. In particular, the authors show that the renormalized perturbative RG equation for the quartic coupling constant incorrectly predicts a flow toward negative values of $\lambda$, which in turn signals an (apparent) instability of the effective potential. However, they also show that, when the finite cutoff is correctly enforced in the effective Higgs-Yukawa theory, in the high energy regime the true flow diverges from the renormalized one. The coupling constant $\lambda$ do not turn to negative values and no instability occurs.

Fig, 4 contains an useful overview of our previous results. When the theory at the scale $\Lambda$ is properly defined, i.e. when $\lambda_{\Lambda}>0$, and $V_{1 l}^{(Y)}(\phi)$ is considered only for values of the field such that $\phi<\Lambda$, no instability occurs.

Before ending this section, it is worth to mention an additional point. In [5] is acknowledged that "the perturbation expansion for $\mathrm{V}$ breaks down" at the point where the non-convexity takes place, that is at the inflection points (see Fig.5 of [5]), which, in their notations, occurs when the "renormalization group time" $t=\ln \mu$ takes the value $t \sim 2.98$. However, they note that for larger values of $t$ their perturbative parameter become again small, so they claim that perturbation theory results can be trusted. From the above analysis (as well as from the results of [2] and [11]), however, is clear that once at a given scale the results of the renormalized perturbation theory start to diverge from the effective theory (bare) ones, this divergence is kept at all higher scales, no matter how small the perturbative parameter is. In other words, the region of instability of $V_{1 l}^{(Y)}(\phi)$ lies well beyond the range of validity of the renormalized perturbative result for $V_{1 l}^{(Y)}(\phi)$ itself.

\section{Summary and conclusions}

In the present work we have shown that, contrary to recent claims [5], the instability of the Higgs-Yukawa one-loop potential $V_{1 l}^{(Y)}(\phi)$ induced by the fermion loop contribution cannot be understood in terms of the very insightful work of Weinberg and $\mathrm{Wu}$ [7], where the physical meaning of the non-convex part of the one-loop scalar effective potential $V_{1 l}^{(s)}(\phi)$ (as well as of its imaginary part) was investigated. We have shown in some

detail that the instability (non-convexity) of the Higgs-Yukawa potential $V_{1 l}^{(Y)}(\phi)$ has a completely different origin from the non-convexity of $V_{1 l}^{(s)}(\phi)$ in the region between the classical minima. 


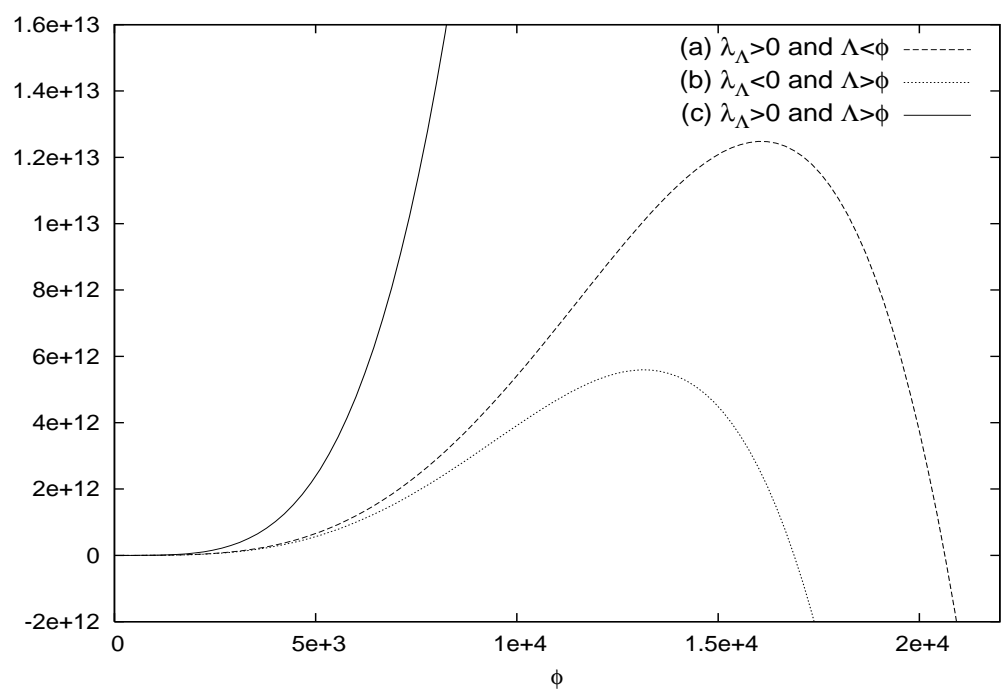

Figure 4: The effective potential $V_{1 l}^{(Y)}(\phi)$ for the three following cases (arbitrary units): (a) $\Lambda=5 \cdot 10^{2}, m_{\Lambda}^{2}=-5 \cdot 10^{2}, \lambda_{\Lambda}=5 \cdot 10^{-2}$. The potential becomes unstable for values of the field $\phi>\Lambda$; (b) $\Lambda=2 \cdot 10^{4}, m_{\Lambda}^{2}=-5 \cdot 10^{2}, \lambda_{\Lambda}=-5 \cdot 10^{-2}$. The potential becomes unstable for values of the field $\phi<\Lambda$. However, being $\lambda_{\Lambda}<0$, the bare potential is unbounded from below and the theory is not defined. (c) $\Lambda=2 \cdot 10^{4}, m_{\Lambda}^{2}=-5 \cdot 10^{2}$, $\lambda_{\Lambda}=5 \cdot 10^{-2}$. The potential is convex in the external region. In the internal region, as we know, the saddle point competition produces a flat potential (Maxwell construction).

Contrary to this latter case, where a physical meaning to the non-convex $V_{1 l}^{(s)}(\phi)$ can be given [7], the instability induced by the fermion loop in the Higgs-Yukawa potential results from an illegal extrapolation of $V_{1 l}^{(Y)}(\phi)$ itself beyond its region of validity. From a renormalization group point of view, the instability comes from the fact that, for high energy scales, the RG equations apparently drive the quartic coupling constant $\lambda$ to negative values (see, for instance, [11, [5]). As we have shown, however, the perturbative RG equations are no longer valid at these scales (see also [11]).

In this respect, it is worth to say a word of caution about the dimensional regularization scheme. This scheme, which is very powerful to get the finite results of renormalized perturbation theory, can be safely used only when the latter holds. One of the most striking drawbacks of the method, which has some resemblance with our problem, concerns the Appelquist-Carazzone decoupling theorem [12]. Being mass-independent, the $\overline{M S}$ scheme cannot reproduce the results of this theorem (see, for instance, [13]). Another, largely unnoticed, drawback concerns the instability problem. As we have seen, the instability of the effective potential occurs for values of $\phi$ beyond the physical cutoff. This can be detected only when a more physical scheme, as a momentum cutoff, is adopted. When this is done, the illusory nature of the effective potential instability becomes immediately clear (see also 2] and [1]).

Finally, it is worth to note that the instability problem is of theoretical as well as of 
phenomenological interest. On the theoretical side, we believe that our analysis shows that, in general, fermion corrections as the ones occurring in the one-loop potential of the Higgs-Yukawa model, the top loop in the case of the SM, cannot destabilize the classical vacuum. On the phenomenological side, it is well known that the most important application concerns the lower bounds on the Higgs boson mass. Our analysis shows that the traditional way of setting these bounds does not work. In [2] and [11, however, novel methods for extracting lower bounds for the Higgs mass have been put forward.

\section{References}

[1] N. Cabibbo, L. Maiani, G. Parisi, R. Petronzio, Nucl.Phys. B158 (1979) 295; R.A. Flores, M. Sher, Phys. Rev. D27 (1983) 1679; M. Lindner, Z. Phys. 31 (1986) 295; M. Sher, Phys. Rep. 179 (1989) 273; M. Lindner, M. Sher, H. W. Zaglauer, Phys. Lett. B228 (1989) 139; C. Ford, D.R.T. Jones, P.W. Stephenson, M.B. Einhorn, Nucl.Phys. B395 (1993) 17; M. Sher, Phys. Lett. B317 (1993) 159; G. Altarelli, G. Isidori, Phys. Lett. B337 (1994) 141; J.A. Casas, J.R. Espinosa, M. Quirós, Phys. Lett. B342 (1995) 171; J.A. Casas, J.R. Espinosa, M. Quirós, Phys. Lett. B382 (1996) 374; G. Isidori, G. Ridolfi, A. Strumia, Nucl. Phys. B609 (2001) 387.

[2] V. Branchina, H. Faivre, Phys. Rev. D 72065017 (2005).

[3] K. Symanzik, Commun. Math. Phys. 16, 48 (1970).

[4] J. Iliopoulos, C. Itzykson, A. Martin, Rev. Mod. Phys. 47, 165 (1975)

[5] M. B. Einhorn, D. R. T. Jones, JHEP 0704:051 (2007).

[6] C. Ford, D.R.T. Jones, P.W. Stephenson, M.B. Einhorn, Nucl. Phys. B395, 17 (1993).

[7] E. J. Weinberg, A. Wu, Phys. Rev. D 36, 2474 (1987).

[8] A. H. Guth, S. Y. Pi, Phys Rev. D 32, 1899 (1985).

[9] P.M. Stevenson, Phys. Rev. D 32, 1389 (1985).

[10] R.J. Rivers, Path Integral Methods in Quantum Field Theory, Cambridge University Press (1987); R.J. Rivers, Z. Phys. C 22, 137 (1984).

[11] Z. Fodor, K. Holland, J. Kuti, D. Nogradi and C. Schroeder, arXiv:0710.3151 [heplat].

[12] T. Appelquist, J. Carazzone, Phys. Rev. D 11, 2856 (1975).

[13] A.V. Manohar, Lectures at Schladming Winter School (1996), hep-th/9606222. 